\title{
MACROSCOPIC BEHAVIOR OF GAS FLOW WITH ADSORPTION THROUGH A FRACTURED POROUS MEDIUM
}

P. Royer ${ }^{1}$, J.L. Auriault ${ }^{1}$ and T. Strzelecki ${ }^{2}$

1. Laboratoire "Sols, Solides, Structures", UJF, INPG, CNRS URA 1511, BP 53X 38041 Grenoble cedex 9, France.

2. Institute of Geotechnics and Hydrotechnics of the Technical University of Wroctaw, Wybrzeże Wypiańskiego 27, 50-370 Wrocław, Poland.

Introduction

A fractured porous medium is a double-porosity medium, i.e. it consists of two interacting porous systems whose permeabilities are very different. One of the two porous structures is associated with the fractures and the other one with the porous matrix. Thus, when looking for a macroscopic equivalent description, three separated scales whose characteristic lengths are very different may be under consideration: the pore-scale, the fracture-scale and the macroscopic scale. Modeling gas flow with adsorption through double-porosity media is of particular interest in mining engineering. Indeed, coal is a multiple-porosity system that naturally contains gas. Gas adsorption within coal skeleton is strongly connected to the occurence of a coal-outburst. To prevent outbursts and also to draw up reliable safety measures, an accurate knowledge of coal-gas system behavior is required. A three-scale homogenization method has been first used in [1] and [2] for the investigation of incompressible fluid flow in a deformable fractured porous medium. The local descriptions at both porescale and fracture-scale are expressed by Navier-Stokes equations. This upscaling method allows to convey the influence of the local effects to the macroscopic level. This technique has been taken up again for modeling gas flow through a rigid fractured porous matrix while considering the strong compressibility of the fluid [3], [4], [5], [6]. Here, the adsorption phenomenon is implemented to gas-flow equations at both local scales through additionnal boundary conditions. Then, the macroscopic behavior is derived via the same three-scale homogenization method. 


\section{Medium under Consideration}

To fit the homogenization method for periodic structures to three-scales problems, the medium is assumed to be doubly periodic. There is no loss of generality by introducing the assumption of periodicity [7]. At the pore scale, consider the medium to be $\Omega$-periodic and its characteristic length to be $l$. The solid and the pores occupy the domains $\Omega_{s}$ and $\Omega_{p}$, respectively, and their common boundary is $\Gamma$ (figure 1). A second periodic porous structure exists at the fracture level, whose period is $\Omega^{\prime}$ and whose characteristic length is $l^{\prime}$, such that $l^{\prime} \gg l$. The porous matrix and the fractures occupy the domains $\Omega_{s p}^{\prime}$ and $\Omega_{f}^{\prime}$, respectively, and their common boundary is noted $\Gamma^{\prime}$ (figure 2). As for the macroscopic characteristic length $l^{\prime \prime}$, it must be chosen such that: $l^{\prime \prime} \gg l^{\prime}$. Therefore, the double-porosity medium shows two separations of scales instead of one in the single-porosity case:

- $\frac{l^{\prime}}{l^{\prime \prime}} \ll 1$ between the fracture scale and the macroscopic level,

- $\frac{l}{l} \ll 1$ between the pore scale and the fracture scale.

The macroscopic behavior of such a medium under fluid flow depends upon the relative order of magnitude between these two scale ratios [1], [2]. The largest coupling effects between the pore-flow and the fracture-flow appear when the scales are equally separated, i.e. when $\frac{l}{l}=O\left(\frac{l^{\prime}}{l^{n}}\right)=O(\varepsilon)[1],[2],[3],[4],[5],[6]$. This is the case under consideration hereafter for the investigation of gas flow with adsorption in a rigid fractured porous medium.

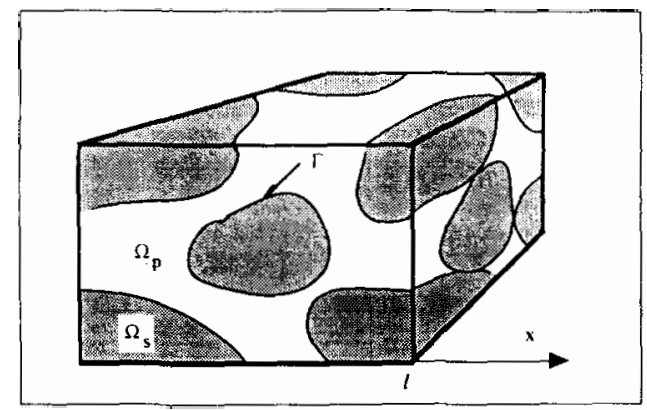

Figure 1: Pore-scale period.

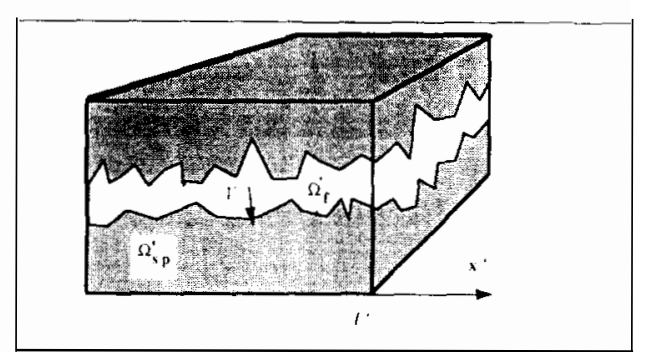

Figure 2: Fracture-scale period. 


\section{Governing Equations}

In the pores $\left(\Omega_{p}\right)$ and in the fractures $\left(\Omega_{f}^{\prime}\right)$, gas flow is expressed by Navier-Stokes equations. The inertial and transient terms of Navier-Stokes equations can be neglected for the first order equivalent macroscopic description [8]. For the sake of simplicity, assume the fluid to be linear. Thus, the gas state equation is a linear relationship between the fluid pressure and the fluid density. As for the adsorption phenomenon, consider the following isotherm linear adsorption law:

$$
(\rho \vec{v}) \cdot \vec{n}=\theta \frac{\partial \rho}{\partial t} \quad \text { on the solid/fluid boundaries, }
$$

where $\theta>0$ and $\vec{n}$ is the exterior normal unit vector of the fluid domain. $\theta$ is the ratio of the adsorbed flux to the time-variation of the density in the neighborhood of the solid/fluid boundary. Therefore, this coefficient is different in the pores and in the fractures. Consequently, two constant coefficients $\theta_{p}$ and $\theta_{f}$ are distinguished. This adsorption law is similar to those used in [9], [10] in the context of diffusion with adsorption so as to model pollutant transport in porous media.

Let us assume the system to be initially at rest: fluid velocity is zero-valued and pressure and density are constant ( $P_{0}$ and $\rho_{0}$, respectively). Thus, the problem is governed by the following set of equations with $k=p$ in the pores $\left(\Omega_{p}\right)$ and $k=f$ in the fractures $\left(\Omega_{f}^{\prime}\right)$. Fluid pressure and density are $P_{0}+P_{k}$ and $\rho_{0}+\rho_{k}$, respectively, where $P_{k}$ and $\rho_{k}$ are increments.

$$
\begin{gathered}
\mu \Delta \vec{v}_{k}+(\lambda+\mu) \vec{\nabla}\left(\vec{\nabla} \cdot \vec{v}_{k}\right)-\vec{\nabla} P_{k}=\overrightarrow{0}, \\
\frac{\partial \rho_{k}}{\partial t}+\vec{\nabla} \cdot\left(\left(\rho_{0}+\rho_{k}\right) \vec{v}_{k}\right)=0 \\
\rho_{k}=A P_{k} \quad \text { where } \frac{\rho_{0}}{P_{0}}=A \text { is a constant, } \\
\left(\left(\rho_{0}+\rho_{p}\right) \vec{v}_{p}\right) \cdot \vec{n}=\theta_{p} \frac{\partial \rho_{p}}{\partial t} \text { on } \Gamma, \\
\left(\left(\rho_{0}+\rho_{f}\right)\left(\vec{v}_{f}-<\vec{v}_{p}>_{\Omega}\right)\right) \cdot \vec{n}^{\prime}=\theta_{f} \frac{\partial \rho_{f}}{\partial t} \text { on } \Gamma^{\prime}, \\
P_{f}=P_{p} \text { on } \Gamma^{\prime}
\end{gathered}
$$




\section{Dimensionless Numbers}

The momentum balance (equation (2)) introduces the following dimensionless number:

$$
Q_{k}=\frac{\left|\vec{\nabla} P_{k}\right|}{\left|\mu \Delta \vec{v}_{k}+(\lambda+\mu) \vec{\nabla}\left(\vec{\nabla} \cdot \vec{v}_{k}\right)\right|}, \quad k=p, f .
$$

It can be shown [4], [5], [6] that $Q=Q_{p}=Q_{f}=O\left(\left(\frac{l^{\prime}}{l^{\prime \prime}}\right)^{-2}\right)=O\left(\varepsilon^{-2}\right)$.

The mass balance (equation (3)) shows the Strouhal number:

$$
S_{k}=\frac{\left|\partial \rho_{k} / \partial t\right|}{\mid \vec{\nabla} \cdot\left(\left(\rho_{0}+\rho_{k}\right) \vec{v}_{k} \mid\right.}, \quad k=p, f .
$$

It has been shown [8] that the case of interest is those where $S_{f}=O(1)$, and that for equally separated scales we have $S_{p}=O(1)$ [5], [6].

The dimensionless number showing adsorption influence (equations (5) and (6)) is:

$$
R_{k}=\frac{\left|\theta_{k} \frac{\partial \rho_{k}}{\partial t}\right|}{\left|\left(\rho_{k} \vec{v}_{k}\right) \cdot \vec{n}\right|}, \quad k=p, f
$$

The case of largest adsorption influence for which the homogenization method can be applied is those where $R_{p}=O\left(\varepsilon^{2}\right)$ and $R_{f}=O(\varepsilon)[6]$. Hence, $\theta_{p}$ and $\theta_{f}$ are assumed to be such that the two above relationships are verified.

Thus, when cast in dimensionless form, the governing equations are written as follows, where all quantities are now dimensionless quantities:

$$
\begin{gathered}
\varepsilon^{2} \mu \Delta \vec{v}_{k}+\varepsilon^{2}(\lambda+\mu) \vec{\nabla}\left(\vec{\nabla} \cdot \vec{v}_{k}\right)-\vec{\nabla} P_{k}=\overrightarrow{0}, \\
\frac{\partial \rho_{k}}{\partial t}+\vec{\nabla} \cdot\left(\left(\rho_{0}+\rho_{k}\right) \vec{v}_{k}\right)=0, \\
\rho_{k}=A P_{k}, \\
\left(\left(\rho_{0}+\rho_{p}\right) \vec{v}_{p}\right) \cdot \vec{n}=\varepsilon^{2} \theta_{p} \frac{\partial \rho_{p}}{\partial t} \text { on } \Gamma, \\
\left(\left(\rho_{0}+\rho_{f}\right)\left(\vec{v}_{f}-<\vec{v}_{p}>_{\Omega}\right)\right) \cdot \vec{n}^{\prime}=\varepsilon \theta_{f} \frac{\partial \rho_{f}}{\partial t} \text { on } \Gamma^{\prime}, \\
P_{f}=P_{p} \text { on } \Gamma^{\prime}
\end{gathered}
$$

The upscaling process may now be performed. Density, pressure and velocity fields are looked for in the form of asymptotic expansions in power of $\varepsilon$. Once these expansions 
have been introduced in the dimensionless set of equations (8-13), the successive boundaryvalue problems at the different orders of $\varepsilon$ must be resolved so as to derive the macroscopic description. Application of homogenization theory to this problem and calculus are detailled in [6]. For a general introduction to the homogenization method for periodic structures, the reader is referred to [7].

\section{Macroscopic Behavior}

The derived macroscopic behavior is:

$$
\left(n^{\prime}+\theta_{f} a_{f}\right) \frac{\partial P_{f}}{\partial t}+\left(n+\theta_{p} a_{p}\right) \frac{<P_{p}>_{e f f}}{\partial t}-\vec{\nabla} \cdot\left[\left(P_{0}+P_{f}\right) \tilde{K}_{f} \vec{\nabla} P_{f}\right]=0
$$

- $a_{f}=\frac{\left|\Gamma_{f}^{\prime}\right|}{\left|\Omega^{\prime}\right|}$ and $a_{p}=\frac{\left|\Gamma_{p}\right|}{|\Omega|} \mid$ are specific surfaces

( $\Gamma_{f}^{\prime}, \Gamma_{p}$ : solid total surfaces in $\Omega^{\prime}$ and $\Omega$, respectively),

- $\tilde{K}_{f}$ is the fracture permeability,

- $\left.<P_{p}>_{e f f}=\frac{1}{\Omega^{\prime}}\right\rceil \int_{\Omega^{\prime} \mathrm{p}} P_{p} d \Omega$,

- $P_{p}$ is $\Omega$-periodic and defined by the following boundary-value problem on $\Omega^{\prime}$ :

$$
\begin{gathered}
\left(n+\theta_{p} a_{p}\right) \frac{\partial P_{p}}{\partial t}-\vec{\nabla} \cdot\left[\left(P_{0}+P_{p}\right) \tilde{K}_{p} \vec{\nabla} P_{p}\right]=0 \\
P_{p}=P_{f} \text { on } \Gamma^{\prime}
\end{gathered}
$$

- $P_{p}=\mathcal{F}\left(P_{f}\right)$, where $\mathcal{F}$ is a nonlinear time-dependent functional exhibiting memory effects.

Concluding Remarks

The macroscopic behavior is strongly influenced by the flow in the pores. It induces memory effects and strong non-linearities. This result highlights how the local effects may affect the macroscopic behavior. This macroscopic description is similar to those obtained in terms of concentrations in [9] to depict pollutant transfer. In this latter paper, the authors take up again some of the notations commonly used in the phenomenological diffusion-adsorption 
investigations, in order to connect the result obtained through homogenization to the concerned literature. Here, two retardation coefficients similar to those used in Soils Mechanics can be defined:

$$
r_{p}=n+\theta_{p} a_{p} \text { in the pores } \quad r_{f}=n^{\prime}+\theta_{f} a_{f} \text { in the fractures }
$$

These coefficients display both filtration transient contribution ( $n$ and $n^{\prime}$ ) and adsorption influence $\left(\theta_{p}, a_{p}, \theta_{f}, a_{f}\right)$.

In this investigation, only the case where $R_{p}=O\left(\varepsilon^{2}\right)$ and $R_{f}=O(\varepsilon)$ has been considered.

This is the case where the homogenization can be applied for which the adsorption influence is the most important. For higher orders of magnitude of these dimensionless numbers, the seepage process is affected by gas adsorption and then the homogenization cannot be applied. Thus, for $R_{p}>O\left(\varepsilon^{2}\right)$ and $R_{f}>O(\varepsilon)$, there is no rigorous equivalent macroscopic description,i.e. independent from sample size and boundary conditions [6]. As for the case in which $R_{p}<O\left(\varepsilon^{2}\right)$ and $R_{f}<O(\varepsilon)$, the first-order macroscopic behavior is no longer influenced by adsorption.

The investigation of porous media with several porosities is of greatest importance in petroleum engineering and mining engineering. The latter concerns in particular the determination of setting of factors contributing to coal-gas outburst occurence. Unless this also depends upon many others phenomena such as absorption and sorption-swelling, the introduction of adsorption in a double-porosity model is an important step in the modeling.

\section{$\underline{\text { References }}$}

1. J.L. Auriault and C. Boutin, TIPM, 7, 63 (1992)

2. J.L. Auriault and C. Boutin, TIPM, 10, 153 (1993)

3. P. Royer and J.L. Auriault, Studia Geotechnica et Mechanica, 13, 65 (1992)

4. J.L. Auriault and P. Royer, C.R.A.S.,II, 431 (1993)

5. P. Royer and J.L. Auriault, TIPM,17, 33 (1994)

6. P. Royer, PhD Diss., Un. J. Fourier, Grenoble, France (1994)

7. J.L. Auriault, Int. J. Eng. Sci., 29, 785 (1991)

8. J.L. Auriault, T. Strzelecki, J. Bauer and S. He, Eur. J. Mech. A/Solids, 9, 373 (1990)

9. J.L. Auriault and J. Lewandowska, J.Geot., 43, 457, (1993)

10. J.L. Auriault and J. Lewandowska, Arch. mech., 45, 51 (1993) 
Acknowledgement

This work has been sponsored by the Polish Scientific Research Committee in Warsaw (Grant KBN No.7S10304305). 